\title{
Intravitreal administration of bevacizumab: pros and cons
}

\author{
Simin Dashti-Khavidaki ${ }^{1}$ and Mohammad Abdollahi ${ }^{2^{*}}$
}

\section{Bevacizumab uses}

Bevacizumab (Avastin', Genethech, Inc.) is a humanized monoclonal antibody that inhibits vascular endothelial growth factor (VEGF). It was first approved by the US food and drug administration for metastatic colorectal cancer and subsequently for cervical/ovarian/fallopian tube cancers, glioblastoma, non-small cell lung cancer, and metastaic renal cell carcinoma [1]. Recently its offlabel use for age-related macular degeneration (AMD) has received extensive attention. Single dose vial of intravitreal ranibizumab (IVR), another anti-VEGF antibody, has been licensed for treatment of wet AMD and diabetic macular edema [1,2]. Due to lower cost and reported equall efficacy and safety data in large clinicat trials such as The Comparison of Age-related Macular Degeneration Treatment Trial (CATT), The Inhibitors of VEGF in Age-related Choroidal Neovascularization (IVAN) and GEFAL studies, intravitreal bevacizumab (IVB) has replaced the intravitreal administration of the approved drug ranibizumab [2-7]. A recent review on IVB administration for management of AMD and pathological myopathy concluded that despite promising results, short-term patients' follow-up in the available studies, lack of well-designed extensive clinical trials, and insufficient data on safety of IVB make its use cautiously in these ocular diseases [8]. IVB administration has been widely used in other different proliferative retinopathies including retinopathy of prematurity $[9,10]$ and diabetic retinopathy $[9,11]$ as well. Angiogenesis with key regulator VEGF is the main pathology in these retinal and choroidal diseases [9]. A review on IVB administration for treatment of different aforementioned retinal and choroidal diseases showed promising reults [12].

\footnotetext{
* Correspondence: mohammad@sina.tums.ac.ir

${ }^{2}$ Faculty of Pharmacy and Pharmaceutical Sciences Research Center, Tehran University of Medical Sciences, Tehran 1417614411, Iran

Full list of author information is available at the end of the article
}

\section{IVB complications}

Two categories of complications have been reported for IVB: post-injection and drug class-related complications. Post-injection complications that are not influenced by the underlying ocular disease include infectious endophthalmitis, sterile intraocular inflammation, rhegmatogenous retinal detachment, increased intraocular pressure and ocular hemorrhage. Drug-related dverse events which are influenced by underlying ocular disease include development or progression of tractional retinal detachment when IVB administration done before vitrectomy in patients with advanced proliferative diabetic retinopathy, possible increase in the incidence of retinal pigment epithelium tears in patients with AMD and worsening retinal detachment in patients with severe retinopathy of prematurity $[9,12]$.

Preservative free vials of bevacizumab have not been created for intravitreal administration.A recent systematic review with aim of evaluating the safety of IVB monotherapy in adult ophthalmic conditions assessed 22 controlled trials and 67 observational studies that contained at least 10 patients showed no difference in the rate of endophthalmitis between the two groups of patients receiving off-labeled IVB or licensed IVR [13]. There are several methods for preparing IVB including multiple withdrawal from the same vial within the same day or at different days under aseptic conditions by ophthalmologist or preparing single use syringes of IVB injection from bevacizumab vials by a compounding pharmacy or a pharmaceutical company. Based on that review,visual loss was the most commonly reported ocular event in patients who received IVB. Nine observational studies reported visual loss with an incidence rate of 0 to $50 \%$. The definition of visual loss was not clear in most of these observational studies. It seems that in these studies, visual loss occasionally was related to other adverse events such as intraocular or anterior chamber inflammation or retinal detachment. Therefore the authors of that review doubted whether visual loss happened due to IVB treatment or progression of the 
patients' diseases. Infectious endophthalmitis was reported in 10 out of 13 observational studies with clinical incidence rate of $0-1 \%$. In three out of 13 studies in which patients were treated with locally prepared IVB,the incidence rates of infectious endophthalmitis were $0.02 \%$, $0.2 \%$, and $0.8 \%$. A higher rate of endophthalmitis $(0.9 \%)$ was reported in the study in which IVB was prepared by a compounding pharmaceutical company [13]. That review concluded that IVB adverse effects are low and comparable with other intravitreal treatments, sham injection, and laser therapy [13].

A case-series from a private ophthalmology center from Hong Kong showed that using the same bevacizumab vial for maximum 10 consecutive injections with multiple aspiration of drug from the same vial under proper sterile technique and discarding remaining drug at the end of the day without overnight storage was safe. No cases of endophthalmitis were reported with IVB from that center [2]. A larger case series from Iran also showed no cluster of endophthalmitis following multiple withdrawal from the same vial within the same day with comparable risk of post-injection endophthalmitis with other intravitreal treatment modalities [14]. Recently, an Indian ophthalmology center reported a cluster of clinically-proven endophthalmitis in 6 out of 8 patients who received IVB by multiple pricks of the same bevacizumab vial (Avastin ${ }^{\circ}$, Genethech, Inc.) by different tuberculin syringes under aseptic precautions but not under the hood. The patients were treated with intravitreal antibiotic administration, but only three patients responded sufficiently. The culture from the bevacizumb vial that had been used for those patients and was kept refrigerated showed no microorganism growth after four days [15].

Artunay et al. reported endophthalmitis incidence rate of $0.066 \%$ after IVB, when multiple doses had been withdrawn from a single vial in an out-patient setting [16]. Other studies have also reported endophthalmitis possibly due to contamination during the compounding procedures of bevacizumab. Yamashiro et al. reported culture-negative endophthalmitis in 14 out of 19 consecutive cases after IVB from a single batch of bevacizumab [17]. Lee et al. reported two patients who received IVB on the same day that developed endophthalmitis with the same strains of Serratia marcescens suggesting contamination during drug compounding. However, they did not find any case of endophthalmitis in another group of patients who received IVB aspirated from the same vial that was reused for multiple consecutive injections just before each procedure and discarded on the same day [18]. Similarly, the Pan-American Collaborative Retina Study Group (PACORES) reported more frequent endophthalmitis in eyes injected using previously compounded aliquots than in eyes given injections from the same multidose vial that was reused appropriately (0.33 vs $0.04 \%)$ [19]. Unexpectedly,a higher frequency of endophthalmitis was reported in patients utilizing compounded aliquots by pharmaceutical company compared to re-utilization of a single vial under aseptic conditions [13]. Another cluster of infectious endophthalmitis with Streptococcus mitis/oralis has been reported in Maiami, Florida, United States in 12 patients. IVB syringes for these patients had been prepared by a compounding pharmacy in South Florida. The same microorganism was found in cultures prepared from unused syringes from this compounding pharmacy [20].

\section{Proposed strategies for IVB preparation}

Although some studies have shown the stability and sterility of compounded bevacizumab at $4{ }^{\circ} \mathrm{C}$ up to 15 days or even 6 months, however, microbial contamination still remains a major concern since bevacizumab vials are preservative free [21,22]. Multiple drawing from the same vial increases the risk of contamination possibly due to wiping rubber septum by fingers or a dirty gauze, rubber stopper leakage, poor aseptic technique (e.g. entering the vial without alcohol swabbing), injection of air into the vial before solution removal, using a contaminated needle or syringe for drug removal, and inappropriate storage durations and temperatures $[23,24]$. An extensive evaluation of IVB prepared by eleven US academic compounding pharmacies under good manufacturing practice facilities showed no microbial or endotoxin contamination. However, there were significant differences in bevacizumab concentrations between prepared aliquots even those prepared in the same compounding pharmacy. Bevacizumab concentrations were lower than those expected in prepared aliquots [25]. Some strategies have been reported to decrease the risk of IVB contamination. One proposed approach to minimize the risk of vial contamination compared to the multiple prick method is to insert a 25 gauge needle into the rubber cap of the vial by single prick and leaving the needle in place. The drug should be drawn into different $1 \mathrm{~mL}$ syringes by every time changing only the syringes not the needle. It is better to use this vial for one day without overnight storage. Each group of called patients should be small in number and for unilateral, not bilateral, IVB $[2,15]$. According to the United States Pharmacopeia (USP), the preparation of IVB aliquots from bevacizumab vials may be categorized to be in medium-risk level for sterile compounding. The quality assurance procedures that recommended to be considered include sterile preparation within a laminarairflow workbench, routine disinfection, air quality testing to reach an International Organization for Standards (ISO) class 5 environment and annual evaluations of 
aseptic manipulation of all pharmacy staffs working in sterile compounding $[2,26]$.

\section{Counterfeit Becacizumab}

Medication counterfeiters are a major threat in drug market all over the world. Expensive drugs such as bevacizumab are at greater risk to be replaced with counterfeiters. Therefore vigilance is needed by physicians, drug distributors and governmental inspection agencies. Detections of counterfeit Avastin in theUnited States [27] and China [28] sparked worldwide concerns. Cheng and Wei have reported an outbreak of acute postoperative endophthalmitis in Shanghai, China after IVB. They mainly incriminated off-label IVB due to the potential risk of endophthalmitis. In their report, 55 out of 116 patients who received IVB from three bevacizumab vials showed endophthalmitis. However, upon testing that bevacizumab batches, Avastin manufacturer and an independent laboratory both showed that there was no bevacizumab in the vials. The cultures of ocular tissues of some of these patients also were negative for any bacteria or fungi. Visual acuities of all these patients were recovered by topical or systemic corticosteroid therapy [28]. Cheng's and Wei's concepts have been criticized by Sun et al. because each expensive drug, including approved IVR is at risk for counterfeiters [29]. Recently happening some adverse effects following IVB treatment in several patients in Iran prompted Ministry of Health and Medical Education of Iran to warn pharmacies of ophthalmology centers to prepare Bevacizumab vials from approved pharmaceutical ditribution companies to be safe from counterfeit bevacizumab (www.ifdona.ir. Lettre number 655/153229 of Food and Drug Organization of Ministry of Health and medical Education of Iran).

\section{Conclusion}

There is growing body of articles on successful use of IVB for the treatment of proliferative retinopathies. Although the off-label use of a drug is not equivalent to off-evidence use of that drug, however, until providing single use, low content vials (1.25 or $2.5 \mathrm{mg})$ of bevacizumab concerns for aseptic conditions and good manufacturing process should be taken to account when preparing aliquots from available preservative free $100 \mathrm{mg} / 4 \mathrm{~mL}$ vials. It is more logical and cautious to perform unilateral not bilateral IVB injection for each patient at each procedur. Additionally, governmental inspection agencies have to be more precise for detection of Bevacizumab counterfeiters.

\section{Competing interests}

The authors declare that they no competing interests.

\section{Author details}

${ }^{1}$ Faculty of Pharmacy, Tehran University of Medical Sciences, Tehran, Iran. ${ }^{2}$ Faculty of Pharmacy and Pharmaceutical Sciences Research Center, Tehran University of Medical Sciences, Tehran 1417614411, Iran.

Received: 26 March 2015 Accepted: 11 April 2015

Published online: 21 April 2015

\section{References}

1. Lexicomp. http://www.lexi.com/.

2. Ng DS, Kwok AK, Chan CW, Li WW. Intravitreal bevacizumab: safety of multiple doses from a single vial for consecutive patients. Hong Kong Med J. 2012;18:488-95.

3. The Comparison of Age-related Macular Degeneration Treatment Trial (CATT) Research Group. Ranibizumab and bevacizumab for neovascular age-related macular degeneration. N Engl J Med. 2011;364:1897-908. doi:10.1056/NEJMoa1102673.

4. The Comparison of Age-related Macular Degeneration Treatment Trial (CATT) Research Group. Ranibizumab and bevacizumab for neovascular age-related macular degeneration.two-year results. Ophthalmology. 2012;119:1388-98. doi:10.1016/j.ophtha.2012.03.053.

5. Meredith TA, McCannel CA, Barr C, Doft BH, Peskin E, Maguire MG, et al. Postinjection endophthalmitis in the comparison of age-related macular degeneration treatment trial (CATT). Ophthalmology. 2015;122:817-21. doi:10.1016/j.ophtha.2014.10.027.

6. Chakravarthy U, Harding SP, Rogers CA, Downes SM, Lotery AJ, Culliford LA, et al. Alternative treatments to inhibit VEGF in age-related choroidal neovascularisation: 2-year finding of the IVAN randomized clinical trial. Lancet. 2013;382:1258-67. doi:10.1016/S0140-6736(13)61501-9.

7. Kodjikian L, Souied EH, Mimoum G, Mauget-Faÿsse M, Behar-Cohen F, Decullier $E$, et al. Ranibizumab versus bevacizumab for neovascular age-related macular degeneration: results from the GEFAL noninferiority randomized trial. Ophthalmology. 2013;120:2300-9. doi:10.1016/ j.ophtha.2013.06.020.

8. Hashemi S, Faramarzi MA, Ghasemi Falavarjani K, Abdollahi M. Bevacizumab for choroidal neovascularization secondary to age-related macular degeneration and pathological myopia. Expert Opin Biol Ther. 2014;14:1837-48. doi:10.1517/14712598.2014.96721.

9. Quiroz-Mercado H. Our experience after 1765 intravitrealinjections of bevacizumab: the importanceof being part of a developing story. Semin Ophthalmol. 2007:22:109-25. doi:10.1080/08820530701420082.

10. Mintz-Hittner HA, Kennedy KA, Chuang AZ, for the BEAT-ROP Cooperative Group. Efficacy of intravitreal bevacizumab for stage 3+ retinopathy of prematurity. New Engl J Med. 2011;364:603-15. doi:10.1056/ NEJMoa1007374.

11. Arevalo JF, Sanchez JG, Lasave AF, Wu L, Maia M, Bonafonte S, et al. Intravitreal bevacizumab (Avastin) for diabetic retinopathy: The 2010 GLADAOF lecture. J Ophthalmol. 2011;2011:584238. doi:10.1155/2011/584238.

12. Falavarjani KG, Nguyen QD. Adverse events and complications associated with intravitreal injection of anti-VEGF agents: a review of literature. Eye. 2013;27:787-94. doi:10.1038/eye.2013.107.

13. Poku E, Rathbone J, Wong R, Everson-Hock E, Essat M, Pandor A, et al. The safety of intravitreal bevacizumab monotherapy in adult ophthalmic conditions: systematic review. BMJ Open. 2014;4:e005244. doi:1.1136/bmjopen-2014-005244.

14. Falavarjani KG, Moddarres M, Hashemi M, Parvaresh MM, Naseripour M, Zare-Moghaddam A, et al. Incidence of acute endophthalmitis after intravitreal bevacizumab injection in a single clinical center. Retina. 2013;33:971-4

15. Khan P, Khan L, Mondal P. Cluster endophthalmitis following multiple intravitreal bevacizumab injections from a single use vial. Indian J Ophthalmol; 2013.

16. Artunay O, Yuzbasioglu E, Rasier R, Sengul A, Bahcecioglu H. Incidence and management of acute endophthalmitis after intravitreal bevacizumab (Avastin) injection. Eye (Lond). 2009;23:2187-93. doi:10.1038/eye.2009.7.

17. Yamashiro K, Tsujikawa A, Miyamoto K, Oh H, Otain A, Tamuara H, et al. Sterile endophthalmitis after intravitreal injection of bevacizumab obtained from a single batch. Retina. 2010;30:485-90. doi:10.1097/IAE.0b013c3181bd2d51. 
18. Lee SH, Woo SJ, Park KH, Kim JH, Song JH, Park KU, et al. Serratia marcescens endophthalmitis associated with intravitreal injections of bevacizumab. Eye (Lond). 2010;24:226-32. doi:10.1038/eye.2009.86.

19. Wu L, Martinez-Castellanos MA, Quiroz-Mercado H, Arevalo JF, Berrocal MH Farah ME, et al. Twelve-month safety of intravitreal injections of bevacizumab (Avastin): results of the Pan-American Collaborative Retina Study Group (PACORES). Graefes Arch Clin Exp Ophthalmol. 2008;246:81-7.

20. Goldberg RA, Flynn Jr HW, Isom R, Miller D, Gonzalez S. An outbreak of Streptococcus endophthalmitis after intravitreal injection of bevacizumab. Am J Ophthalmol. 2012;153:204-8. doi:10.1016/j/ajo.2011.11.035.

21. Chen YH, Wu PC, Shiea J, Lo LH, Wu YC, Kuo HK. Evaluation of the sterility, stability, and efficacy of bevacizumab stored in multiple-dose vials for 6 months. J Ocul Pharmacol Ther. 2009;25:65-9. doi:10.1089/jop2008.0043.

22. Ornek K, Karahan ZC, Ergin A, Tekeli A, Tekeli O. Bevacizumab sterility in multiple doses from a single-use vial. Ann Pharmacother. 2008;42:1425-8. doi:10.1345/aph.1L270.

23. Sheth NK, Post GT, Wisniewski TR, Uttech BV. Multidose vials versus single-dose vials: a study in sterility and cost effectiveness. J Clin Microbiol. 1983;17:377-9.

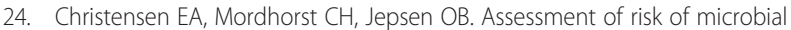
contamination by use of multidose containers of injectable products. J Hosp Infect. 1992;20:301-4.

25. Yannuzzi NA, Klufas MA, Quach L, Beatty LM, Kaminsky SM, Crystal RG, et al. Evaluation of compounded bevacizumab prepared for intravitreal injection. JAMA Ophthalmol. 2015;133:32-9. doi:10.1001/jamaophthalmol.2014.3591.

26. Trissel LA, Gentempo JA, Anderson RW, Lajeunesse JD. Using a medium-fill simulation to evaluate the microbial contamination rate for USP medium-risk level compounding. Am J Health Syst Pharm. 2005;62:285-8.

27. Mackey TK, Cuomo R, Guerra C, Liang BA. After counterfeit avastin ${ }^{\oplus}$-What have we learned and what can be done. Nat Rev Clin Oncol. 2015; doi:10.1038/nrclinonc.2015.35

28. Cheng JW, Wei RL. Ranibizumab for age-related macular degeneration. N Engl J Med. 2011;364:582. doi:10.1056/NEJMc1013316\#SA2.

29. Sun $X$, Xun $X$, Zhang $X$. Counterfeit bevacizumab and endophthalmitis. N Engl J Med. 2011;365:378-9. doi:10.1056/NEJMc1106415\#SA1.

\section{Submit your next manuscript to BioMed Central and take full advantage of:}

- Convenient online submission

- Thorough peer review

- No space constraints or color figure charges

- Immediate publication on acceptance

- Inclusion in PubMed, CAS, Scopus and Google Scholar

- Research which is freely available for redistribution

Submit your manuscript at www.biomedcentral.com/submit 